TITLE:

\title{
Variant and invariant patterns embedded in human locomotion through whole body kinematic coordination.
}

\section{$\operatorname{AUTHOR}(S):$}

Funato, Tetsuro; Aoi, Shinya; Oshima, Hiroko; Tsuchiya, Kazuo

\section{CITATION:}

Funato, Tetsuro ...[et al]. Variant and invariant patterns embedded in human locomotion through whole body kinematic coordination.. Experimental brain research. Experimentelle Hirnforschung. Expérimentation cérébrale 2010, 205(4): 497511

\section{ISSUE DATE:}

2010-09

URL:

http://hdl.handle.net/2433/142025

\section{RIGHT:}

The final publication is available at www.springerlink.com; この論文は 出版社版でありません。引用の際には出版社版をご確認ご利用くださ $\iota_{\circ} ;$ This is not the published version. Please cite only the published version. 


\section{Variant and invariant patterns embedded in human locomotion through whole body kinematic coordination}

Tetsuro Funato, Shinya Aoi, Hiroko Oshima, Kazuo Tsuchiya

T. Funato, Department of Mechanical Engineering and Science, Kyoto University, Yoshida-honmachi, Sakyo-ku, Kyoto 606-8501, Japan

Tel. \& Fax: +81-774-65-6489 funato@me.kyoto-u.ac.jp.

S. Aoi, Department of Aeronautics and Astronautics, Kyoto University, Yoshidahonmachi, Sakyo-ku, Kyoto 606-8501, Japan.

H. Oshima, K. Tsuchiya, Department of Energy and Mechanical Engineering, Doshisha University, 1-3 Tatara-miyakodani, Kyotanabe-shi, Kyoto 610-0321, Japan

Received: date / Accepted: date

Abstract Step length, cadence and joint flexion all increase in response to increases in gradient and walking speed. However, the tuning strategy leading to these changes has not been elucidated. One characteristic of joint variation that occurs during walking is the close relationship among the joints. This property reduces the number of degrees of freedom, and seems to be a key issue in discussing the tuning strategy. This correlation has been analyzed for the lower limbs, but the relation between the trunk and lower body is generally ignored. Two questions about posture during walking are discussed in this paper: (1) whether there is a low-dimensional restriction that determines walking posture, which depends not just on the lower limbs but on the whole body, including the trunk; and (2) whether some simple rules appear in different walking conditions. To investigate the correlation, singular value decomposition was applied to a measured walking pattern. This showed that the whole movement can be described by a closed loop on a twodimensional plane in joint space. Furthermore, by investigating the effect of the walking condition on the decomposed patterns, the position and the tilt of the constraint plane was found to change significantly while the loop pattern on the constraint plane was shown to be robust. This result indicates that humans select only certain kinematic characteristics for adapting to various walking conditions.

Keywords Human locomotion, Walking condition, Slope, Intersegmental coordination

\section{Introduction}

Humans walk on different slopes and with different walking speeds by tuning their whole body motion. However, the tuning parameters and the tuning strategy remain unclear. An increase in walking length and step rate and a decrease in duty-rate have been reported as responses to an increase in walking velocity 
(Milner and Quanbury 1970; Murray et al. 1984; Winter 1987; Stoquart et al. 2008). Higher levels and longer duration of EMG activation have been reported as collateral effects of increases in velocity and slope (Murray et al. 1984; Stoquart et al. 2008; Lay et al. 2007). Some research indicates that step length and cadence change depending on the slope of the surface (Leroux et al. 2002) while other research indicates that these properties are robust against changes of incline (Lay et al. 2006). The difference between these results may be caused by different walking speeds (Yamazaki et al. 1984).

Change in elevation angles is a common characteristic which originates in gradient and speed variation. As the gradient increases, the flexion in the lower limbs at step landing time increases (Lay et al. 2006; Leroux et al. 2002), the amplitude of the motion of trunk and pelvis increases (Murray et al. 1984; Lay et al. 2006), and eventually the body bends forward more deeply (Leroux et al. 2002; Vogt and Banzer 1999). Some researchers contend that such body leaning is a result of tuning the center of mass (COM) position (Leroux et al. 2002), which is based on a report that the COM position is moved forward during walking (Iida and Yamamuro 1987). Grasso et al. compared walking in three postures: normal walking, walking with knee flexion and walking with knee and hip flexion (Grasso et al. 2000). Although an increase in the electromyography (EMG) level and variation in the activation pattern of every muscle were observed with body flexion, the movement patterns of the joints did not change. Furthermore, in the same research, a correlated motion of the lower three limbs was reported: all limbs moved as if constrained to lie on a two-dimensional plane in joint space, and the change in movement corresponding to change of posture can be expressed as a change in the orientation of this constraint plane.

Relationships among the movements of joints have been found in many studies. One that measured joint movement for 108 subjects (Crosbie and Vachalathiti 1997) demonstrated a high correlation between hip flexion and pelvic list independent of age and gender. Other reports have shown correlations between trunk, neck and head (Cromwell 2003) or between the lower limbs (Borghese et al. 1996). Taking all these studies together, movement correlations between all parts of the body have been reported. Correlation among joint movements has been observed and researched not only for walking but also for other whole body motions, like voluntary sway (Alexandrov et al. 1998; Martin et 
al. 1999, 2000) and reaching movements (Thomas et al. 2005; Berret et al. 2009). These studies considered all joints, including those of the trunk.

In order to investigate detailed characteristics of these correlations, principal component analysis (PCA) is frequently used. For human walking, two principal components can be derived from the movement of thigh, shank and foot, and these two components create a two-dimensional constraint plane for joint movement (Borghese et al. 1996; Mah et al. 1994). In addition to the previously described postural change, it has been shown by many groups that gradient and walking speed change the tilt of the constraint plane (Bianchi et al. 1998; Noble and Prentice 2008; Ivanenko et al. 2008), and the level of tilt is thought to be determined by the energy required for the movement (Bianchi et al. 1998).

The notion that the movements of the lower limbs are constrained on a correlation plane and that the effects of walking conditions are integrated into a change of that plane (Grasso et al. 2000; Bianchi et al. 1998; Noble and Prentice 2008; Ivanenko et al. 2008) leads to the idea that the many joints in the lower limbs are tuned together. At the same time, it leads to the idea that humans tune only a selected property in response to variations in the walking condition. However, the existence of such a robust property has not been confirmed statistically, and such confirmation is the primary aim of this research. Another unsolved issue is the incorporation of trunk movement. Although the importance of trunk movement for walking has been emphasized in many studies (Sartor et al. 1999; Frigo et al. 2003; Anders et al. 2006), research has focused on the lower limbs (Borghese et al. 1996; Mah et al. 1994; Ivanenko et al. 2007; Onge and Feldman 2003), and the effect of trunk angle in the joint correlation has rarely been discussed (except, for instance, by Courtine and Schieppati (2004)). As mentioned above, joint movements in the upper body and lower limbs are closely correlated, so the existence of constraint spaces for whole body movement is expected, as in the case of the lower limbs. This enables us to bring the COM into the discussion, and to consider whether correlation in whole body movement is important for the tuning strategy.

In this paper, we consider the tuning strategy for the walking condition. We analyze the principal components of the whole body movement during walking, and discuss the variation of these components with changes of slope and walking speed. By performing singular value decomposition (SVD) on the time series of 
joint motion, the motion is decomposed into principal groups of simultaneously active joints (intersegmental coordination) and the activation pattern (temporal coordination) of the joint group. The tuning parameter is distilled by testing the effect of the walking condition on each intersegmental and temporal coordination. The SVD revealed that the movements of the seven measured joints are composed of only two principal components, just as in the case of lower limb motion (Bianchi et al. 1998; Noble and Prentice 2008; Ivanenko et al. 2008). By considering the effect of the walking condition on the extracted constraint plane, we show that the coordination pattern composed of the different postures under various walking conditions can be classified into variant (environment dependent) and invariant patterns.

\section{Materials and Methods}

\section{Experimental procedure}

The subjects were 10 healthy males with an age range of $21-25$, weights of 55$72[\mathrm{~kg}]$ and heights of $165-177[\mathrm{~cm}]$. They walked on a treadmill and the motions of their joints were measured with a motion capturing system (Digital RealTime System manufactured by MotionAnalysis). Reflective markers were attached to the subjects' skin overlying the following body landmarks of both hemibodies: ear tragus, upper limit of the acromion, greater trochanter, lateral condyle of the knee, lateral malleolus, second metatarsal head, and heel. We have focused on the motion of the joints in the sagittal plane, so the makers are placed to facilitate measurements of the motion in that plane.

In this research, motion during the double support (DS) phase and the single support (SS) phase are analyzed independently as described in the next section. The duration of the double support phase is short, so the desired sampling rate is higher than usual. For a standard walking cycle, DS lasts approximately $1.0[\mathrm{~s}]$ in normal gait (Murray et al. 1964) giving 60 data points at the usual $60[\mathrm{~Hz}]$ sampling rate. When we set the fastest walking speed at $5[\mathrm{~km} / \mathrm{h}]$, the duration of DS was approximately $0.2[\mathrm{~s}]$ at pre-test. At a $60[\mathrm{~Hz}]$ sampling rate, the motion in DS is separated into 12 points, which seems to lead to loss of some information about the motion. Therefore, we set the sampling frequency at $300[\mathrm{~Hz}]$, so that the motion is recorded at 60 points. 
In order to focus on involuntary action, the ranges of conditions are selected to avoid inducing an extreme transformation caused by conscious control. By progressively increasing the gradient of the slope and observing the angle of the trunk, an apparent change is detected at about 12[\%], and the subjects feel a strong effect of the slope afterwards. Thus, we set the maximum slope at this level, and the interval is discretized into seven conditions: level ground, $2 \%$ (1.15[ deg ]), 4\% (2.29[ deg ]), 6\% (3.43[ deg ]), 8\% (4.57[ deg ]), 10\% (5.71[ deg ]) and $12 \%(6.84[\mathrm{deg}])$. Standard walking velocity is $3-4[\mathrm{~km} / \mathrm{h}]$, and approximately the same level of trunk inclination observed for various inclines at $3[\mathrm{~km} / \mathrm{h}]$ was observed between $3[\mathrm{~km} / \mathrm{h}]$ and $5[\mathrm{~km} / \mathrm{h}]$ on level ground. So, the velocity condition was set at three levels: $3.0[\mathrm{~km} / \mathrm{h}], 4.0[\mathrm{~km} / \mathrm{h}]$ and $5.0[\mathrm{~km} / \mathrm{h}]$. Recording started after the subjects had been walking on the treadmill for sufficient time for their movement to settle into a regular pattern, and the duration for a record of one walking condition was $15[\mathrm{~s}]$.

In order to analyze the whole body movement without considering the motion of arms, the arms must be fixed at a certain position. The subjects were requested to look forward and place their hands on their waists. This position of the arms was selected to include them with the trunk on the sagittal plane.

Subjects gave informed consent prior to data collection according to the procedures of the Ethics Committee of Doshisha University.

\section{Data preparation}

From the recorded time series of joint positions, we calculated the seven angles of segments defined on the sagittal plane as shown in Fig. 1(i). Every angle was defined as an elevation angle based on the assumption that the elevation angles behave more stereotypically than the relative angles (Borghese et al. 1996; Ivanenko et al. 2007).

We separated the measurements into the double support (DS) phase and the single support (SS) phase because the phases have different dynamic constraints: DS requires the distance between the right and left legs to be fixed. This difference in constraint will strongly affect the motion and might prevent us from clearly interpreting the effect of a condition if we did not make the separation. 
The DS phase was defined as the time from one heel grounding to the toes of the other foot lifting off. The SS phase started at this time and ended with the next heel grounding. Every locomotion cycle was taken to consist of two DS phases and two SS phases. Cycles not fitting that pattern were rejected as outliers. Moreover, the number of data points in one phase was standardized at 300 . If the number of points in the recorded data was insufficient the extra points were constructed using cubic spline interpolation. Figure 1(ii) shows the obtained time series of thigh, shank and foot angles. The four phases are labeled DS1 (starting with right foot contact), SS (supported on right leg), DS2 (starting from left foot contact) and SW (supported on left leg). Because DS1 and DS2 (or SS and SW) are the same except for the difference of right or left, we discuss only DS1 and SS in this paper.

\section{Extraction of the principal movements by SVD}

By writing the time series data of the angles of each joint as a column, the whole movement can be expressed in terms of the matrix

$$
R(\theta, t)=\underset{\downarrow}{\text { time }}\left[\begin{array}{ccc}
\theta_{1}\left(t_{1}\right), & \theta_{2}\left(t_{1}\right), & \cdots \\
\theta_{1}\left(t_{2}\right), & \theta_{2}\left(t_{2}\right), & \cdots \\
\vdots &
\end{array}\right]
$$

where $\theta_{i}\left(t_{\mathrm{j}}\right)$ is the angle for joint $i$ at the $j$-th point in the time series. Thus, a vector drawn from one row of the $300 \times 7$ matrix $R$ is a set of elevation angles at a particular time, and a vector drawn from one column of $R$ is a time series for a particular joint.

The whole body motion is decomposed into the time-invariant coordination of segments and its time-series gain, by extracting a highly correlated group of joints from $R(\theta, t)=R_{0}(\theta)+V(t) \cdot \Lambda \cdot Z^{T}(\theta)$, as follows.

1. Principal component analysis is applied to the covariance matrix $\operatorname{cov}(R(\theta))$ with elevation angles $\theta$ as variables. The principal components $z_{i}$ are the vectors with a high correlation among joints and describe intersegmental coordination.

2. The time averages of the elevation angles are written as 
$r_{0}(\theta)=\left[\overline{\theta_{1}} \cdots \overline{\theta_{7}}\right]^{T}$. Then, $r_{0}$ represents one posture in the phase, called the "mean posture" in this paper. The matrix $R_{0}(\theta)$ is constructed by repeating the row $r_{0}^{T} 300$ times.

3. The covariance matrix $\operatorname{cov}(R)$ is a matrix whose $i j$ element is defined by $E\left(\left(\theta_{i}-\bar{\theta}_{i}\right)\left(\theta_{j}-\overline{\theta_{j}}\right)\right)$, and it can be written as $\operatorname{cov}(R)=\frac{1}{7}\left(R-R_{0}\right)^{T}\left(R-R_{0}\right)$. Then, the eigenvectors of $\operatorname{cov}(R), z_{i}$, can be obtained from the right singular vectors of the singular value decomposition of $R-R_{0}$.

$$
\begin{aligned}
R(\theta, t)-R_{0}(\theta) & =V(t) \Lambda Z^{T}(\theta) \\
& =\sum_{i} \lambda_{i} v_{i}(t) z_{i}(\theta) .
\end{aligned}
$$

Here $\lambda_{i} v_{i}(t)$ denotes the (time series) values with the basis $z_{i}(\theta)$. $\lambda_{i} v_{i}(t)$ is the coordination pattern of temporal characteristics of the motion corresponding to $z_{i}$, so $\lambda_{i} v_{i}$ is referred to as the temporal coordination in this paper.

Thus, by performing SVD on $R(\theta, t)$, whole body motion is decomposed into intersegmental coordination $z_{i}$, temporal coordination $\lambda_{i} v_{i}$ and mean posture $r_{0}\left(R_{0}\right)$.

$$
R(\theta, t)=R_{0}(\theta)+\sum_{i} \lambda_{i} v_{i}(t) z_{i}^{T}(\theta)
$$

Furthermore, $r_{0}$ can be decomposed into a normalized part, $\left\langle r_{0}\right\rangle$, and a gain, $\left\|r_{0}\right\|$. Then, $r_{0}=\left\|r_{0}\right\| \cdot<r_{0}>$, where $<r_{0}>$ indicates the intersegmental coordination of the mean posture and $\left\|r_{0}\right\|$ indicates the bending level. In addition, $\left\langle r_{0}\right\rangle$ and $\left\|r_{0}\right\|$ correspond to the intersegmental coordination $z_{i}$ and the singular value $\lambda_{i}$ of the movement components.

\section{Statistical methods for pattern similarity}

In order to measure the effect of walking condition on the walking pattern obtained by SVD, a statistical method for determining similarity is applied to the patterns recorded under different conditions. The intersegmental coordination ( $z$ and $\left\langle r_{0}>\right.$ ), the temporal coordination $(v)$, and the singular values ( $\lambda$ and 
$\left.\left\|r_{0}\right\|\right)$ have different statistical natures, being categorized, respectively, as multivariate nominal, time series and single variables. Thus, each pattern requires a different statistical procedure.

The correlation coefficient is a quantitative measure for the similarity of intersegmental coordination under different conditions. The correlation coefficient can be calculated using the inner product of the pattern vectors which are obtained as normalized vectors by SVD. The significance of the difference between patterns is further verified using 2-way ANOVA of the joints and environmental factors. The temporal coordination is analyzed using two types of test, each focusing on one of the two parts of the pattern. A Kruskal-Wallis rank test determines whether the average distribution changes depending on the conditions. A 2-way ANOVA with temporal and environmental factors is used to detect patterns at some point in time picked up from the whole stance. For the singular values $\lambda$ and $\left\|r_{0}\right\|$, a 1-way ANOVA can be applied directly.

The effect of walking condition on the mean posture is further evaluated by the difference in the mean posture $\Delta r_{0}^{k} . \Delta r_{0}^{k}$ is the difference of the mean posture $r_{0}$ obtained in conditions $k-1$ and $k$,

$$
\Delta r_{0}^{k}=E_{i}\left(\left\|r_{0}^{k_{i}}-E_{j}\left(r_{0}^{(k-1)_{j}}\right)\right\|\right),
$$

where $r_{0}^{k_{i}}$ is mean posture of the $i$ th step recorded in condition $k, E_{i}$ is the mean over all possible steps $i$, and $k$ and $k-1$ are limited to adjacent conditions, such as $2[\%]$ with $4[\%]$ or $4[\%]$ with $6[\%]$. We test whether the mean posture changes at different rates among joints, using 2-way ANOVA on $\Delta r_{0}^{k}$ with joint and environmental factors. 


\section{Results}

\section{Principal segmental and temporal coordination derived by SVD}

\section{Coordination patterns obtained by SVD}

The joint motion was recorded for 10 subjects and the matrices $R(\theta, t)$ were constructed. The coordination patterns $\lambda_{i} v_{i}$ and $z_{i}$ were obtained by the SVD $R(\theta, t)=R_{0}(\theta)+\sum_{i} \lambda_{i} v_{i}(t) z_{i}^{T}(\theta)$. The singular value $\lambda$ and the cumulative

proportions $\sum_{i=1}^{j} \lambda_{i}^{2} / \sum_{i=1}^{7} \lambda_{i}^{2}$ computed through the SVD are listed in Table 1(i). When DS and SS are treated separately, the cumulative proportion exceeds $99 \%$ by the second coordination for every subject, which indicates that the whole body motion can be represented as the sum of only two movements. In addition, the SVD decomposes the two movements into the intersegmental coordinations, $\left(z_{1}, z_{2}\right)$, and the temporal coordinations, $\left(\lambda_{1} v_{1}, \lambda_{2} v_{2}\right)$, as shown in Fig. 2.

\section{Walking motion in joint space}

By SVD, we found that the motion of DS and SS phases can each be described by two intersegmental coordinations $\left(z_{1}, z_{2}\right)$ and two temporal coordinations $\left(\lambda_{1} v_{1}, \lambda_{2} v_{2}\right)$. This result indicates that the motion of each support phase is constrained on a plane having $z_{1}$ and $z_{2}$ as its axes. Because whole motion is composed of DS and SS phases, there are two constraint planes $\left(z_{1}^{\mathrm{DS}}, z_{2}^{\mathrm{DS}}\right)$ and $\left(z_{1}^{\mathrm{SS}}, z_{2}^{\mathrm{SS}}\right)$, where both planes are sub-spaces of seven-dimensional joint space. Because the motion switches between DS and SS, the two spaces intersect at the switching points.

In order to consider the space of the whole motion including both DS and SS (DS-SS), SVD is applied to the motion of one half of a step cycle (DS1 and SS, from the right heel grounding to the left heel grounding). The singular value and the cumulative proportions are listed in Table 1(ii). The third singular value of DS-SS is between the second singular value of DS and second singular value of SS (Table 1(i)), and the cumulative proportion of DS-SS exceeds 99[\%] after three elements. This result indicates that the whole motion including DS and SS is 
included in the space spanned by three intersegmental coordinations. We call the three intersegmental coordinations $\tilde{z}_{1}, \tilde{z}_{2}$ and $\tilde{z}_{3}$. Then, the space having $\tilde{z}_{1}, \tilde{z}_{2}, \tilde{z}_{3}$ as its axes includes the motion of DS and SS and the constraint planes derived by treating DS and SS separately. Figure 3 shows the walking motion in the three-dimensional space spanned by $\tilde{z}_{1}, \tilde{z}_{2}$ and $\tilde{z}_{3}$ (the projection of coordinate values is described in detail in the Appendix). The example path shown in Fig. 3(i) is the measured posture of one cycle and the two planes are the constraint planes for the DS and SS phases. By assuming left-right symmetry of the body, the end point of SS and the initial point of DS can be regarded as being identical, although they are located at different positions in the space. Thus, by jumping from the end of SS to the initial position of DS, the state of the walking motion describes a closed loop in the assumed space.

The constraint planes calculated from the motion on different slopes $(0,4,8$, 12[\%]) are shown in Fig. 3(ii). The figure shows that the tilt of the plane changes as the walking condition changes, but these changes are not large compared to the difference between the DS and SS planes.

\section{Effect of walking condition on the patterns}

By describing the whole body movement as a closed loop on two planes (as shown in Fig. 3), the nature of walking is determined by four characteristics: the tilts of the planes in the seven-dimensional joint space, which is determined by $z_{1}$ and $z_{2}$; the shape and size of the closed loop, corresponding to $\lambda_{1} v_{1}$ and $\lambda_{2} v_{2}$; the location of the origin of the $z_{1}-z_{2}$ plane, $r_{0}$; and the cadence. The last characteristic is not considered in this paper.

In order to clarify the effect of different walking environments on these characteristics, we investigated the environment dependency of the following patterns.

- Temporal coordination: $\left(\lambda_{1} v_{1}, \lambda_{2} v_{2}\right)$

- Intersegmental coordination: $\left(z_{1}, z_{2}\right)$

- Mean posture: $\left\|r_{0}\right\|$ and $<r_{0}>$

The tilt of constraint planes is further analyzed by comparing normal vectors in addition to $z_{1}$ and $z_{2}$. 
Temporal coordination: $\left(\lambda_{1} v_{1}, \lambda_{2} v_{2}\right)$

The temporal coordination is averaged for 14 steps recorded under each of the three different velocities $(3[\mathrm{~km} / \mathrm{h}], 4[\mathrm{~km} / \mathrm{h}]$ and $5[\mathrm{~km} / \mathrm{h}])$. Figure $4(\mathrm{i})$ shows the high similarity of the averaged patterns. In order to find any differences among the patterns exhibited under the different velocity conditions, two different tests were performed. The first test deals with differences among average distributions (Fig. 4(i)), and the second test discusses the independence of the average distribution in $10 \%$ segments of the support phase using the variance at the appropriate time. For the first test, a Shapiro-Wilk normality test gives $p<1.0 \times 10^{-5}$ for every pattern, so a Kruskal-Wallis rank test is applied. The results, listed in Table 2(i), show no significant effect of velocity at the 5\% level. The discretized patterns were compared using 2-way ANOVA with factors time and velocity. The p-values of the velocity factor, given in Table 3(i), show no significant effect of velocity on the patterns apart from in 2 subjects.

Turning our attention to the effect of the incline, the average temporal coordinations for different slopes are shown in Fig. 4(ii). There are no obvious differences between the patterns, and a Kruskal-Wallis rank test found no significant effect of incline (see Table 2(ii)). Applying a 2-way ANOVA with factors time and gradient to the discretized distribution indicates that no more than 2 subjects show a significant effect of gradient (see Table 3(ii)). Thus, temporal coordination was demonstrated to be robust against changes in incline.

Intersegmental coordination: $\left(z_{1}, z_{2}\right)$

A typical intersegmental coordination $\left(z_{1}, z_{2}\right)$ computed for speeds of $3[\mathrm{~km} / \mathrm{h}]$, $4[\mathrm{~km} / \mathrm{h}]$ and $5[\mathrm{~km} / \mathrm{h}]$ is shown in Fig. 5(i). The figure shows a similar shape at each velocity, and the high similarity is also supported by the correlation coefficients shown in Table 4(i). The p-values of the velocity factor for a 2-way ANOVA with factors joint and velocity are given in Table 5(i) which shows that about half of the subjects exhibit a statistically significant effect of velocity on intersegmental coordination at the $1 \%$ level.

The intersegmental coordinations for different inclines are shown in Fig. 5(ii). Although the shape of the patterns in the figure and the correlation coefficients listed in Table 4(ii) indicate the high similarity of the patterns, a gradual change 
with incline can be seen, particularly in $z_{2}$ (see Fig. 5(ii)). In order to determine the statistical significance of this effect of slope, a 2-way ANOVA with factors joint and gradient is performed. Table 5(ii) gives p-values of the gradient factor. This table shows that, for all subjects, there is a significant effect of incline on $z_{2}$ in SS; there is also a significant effect on the other coordination for most subjects.

\section{Mean posture: $r_{0}$}

To consider the effect of walking condition on the posture averaged for the support phase, $r_{0}$, the posture was decomposed into the normalized vector $\left.<r_{0}\right\rangle$ and the gain $\left\|r_{0}\right\| .<r_{0}>$ and $\left\|r_{0}\right\|$ for different velocities are shown in Fig. 6(i) and Fig. 7(i). Figure 7(i) shows the apparent growth of $\left\|r_{0}\right\|$ with an increase in velocity. The effect of velocity was examined using a 1-way ANOVA for $\left\|r_{0}\right\|$ and a 2-way ANOVA with factors joints and velocities for $\left\langle r_{0}\right\rangle$. Statistically significant differences were found at the $1 \%$ level in $\left\|r_{0}\right\|$ for all subjects and in $\left\langle r_{0}\right\rangle$ for 8 subjects.

Values for $\left\langle r_{0}>\right.$ and $\left\|r_{0}\right\|$ on various inclines are shown in Fig. 6(ii) and Fig. 7(ii). These figures show the growth of $\left\|r_{0}\right\|$ with an increase in the gradient. A 1-way ANOVA for $\left\|r_{0}\right\|$ and a 2-way ANOVA for $<r_{0}>$ with factors joints and inclines found statistically significant differences (at the 1\% level) in both $\left\langle r_{0}>\right.$ and $\left\|r_{0}\right\|$ for all subjects.

The above result indicates that the variation in the mean posture is obvious. In order to study the change in each elevation angle of mean posture, the difference in mean posture among conditions $\Delta r_{0}^{k}$ was calculated for three walking velocities and seven slope conditions (see Fig. 8). No large differences among joints were found. In order to study whether or not the mean posture changed at different rates among joints, the differences among each joint element of $\Delta r_{0}^{k}$ were tested with a 2-way ANOVA with factors of joint and walking condition. The level of variation is significantly different among joints $(p<0.01$ for both DS and SS, in all velocity and slope conditions). The most variable joints in both DS and SS are the foot of lift-off and the swing leg. The difference between the foot and the other joints is clearer in DS than SS. Although statistical uniformity 
among joints was rejected, the similarity of the level among joints is not negligible. In particular, although the range of trunk motion is rather small compared to the other angles and a difference in the level of trunk is apparent in both mean posture and intersegmental coordination, the variation of the trunk in the mean posture is not extremely small compared to the other joints.

In summary, the mean posture $r_{0}$ was affected by changes in walking condition. In particular, $\left\|r_{0}\right\|$ grew with both increasing velocity and increasing incline. Variation in the mean posture occurs primarily in the foot of lift-off and swing legs, although the other joints also exhibit a certain degree of change.

\section{Normal vectors of constraint planes}

Whereas $z_{1}$ and $z_{2}$ were evaluated independently in the previous section, it is possible to evaluate the intersegmental coordination under different conditions as a property of the constraint planes. In particular, when the constraint planes are embedded in a three-dimensional subspace of the seven-dimensional joint space, their tilt is characterized by one normal vector. Thus the relationship between planes can be quantitatively evaluated by calculating the angles between normal vectors. The relative angles between the normal vectors for level ground and those for each gradient are shown in Fig. 9(i). Here, the relative angle between vectors $a$ and $b$ is $\frac{180}{\pi} \cos ^{-1}\left(\frac{a \cdot b}{\|a\|\|b\|}\right)$ [deg]. The figure shows that the relative angles increase with an increase in the gradient, although the standard deviation is large because the values are rather different among subjects. In order to compute the relative tilt of the constraint plane for different conditions while removing the effects of differences among subjects, the rank of the relative angles for each subject is calculated. Fig. 9(ii) shows the rank of each gradient condition summarized by subject and steps. The rank, that is, the relative tilt of the constraint planes increases with an increase in the gradient. Testing the difference of the rank among conditions using 1-way ANOVA shows that the tilt of the constraint plane, which is defined by the intersegmental coordination, depends on the walking condition $(p<0.01)$. 


\section{Discussion}

In this research, seven link-joint movements were measured during walking. The time series of the elevation angles were decomposed by SVD after being separated into double and single support phases. The whole movement was revealed to be composed of a mean posture and two principal movements which were also divided into two intersegmental coordinations and two temporal coordinations. In order to discuss the relationship between the walking condition and the joint motions described as decomposed patterns, the consistency of the patterns among different conditions was verified.

The whole body movement can be described by a closed loop on two constraint planes. The spatial nature of the walking motion is characterized by the location and tilt of the constraint planes, and the temporal nature is characterized by the trajectory on the planes. The position and tilt of the planes is determined by mean posture $\left(r_{0}\right)$ and intersegmental coordination $\left(z_{1}, z_{2}\right)$, respectively, and the trajectory is determined by temporal coordination $\left(\lambda_{1} v_{1}, \lambda_{2} v_{2}\right)$. We now discuss whether each characteristic depends on the walking condition; in other words, whether each characteristic is used as a tuning parameter for adapting to walking conditions.

\section{Effect of walking conditions}

\section{Is the trajectory on the constraint plane affected?}

By considering the similarity of temporal coordination calculated from the recorded data under different walking conditions, both the average distribution and the discretized time period data showed no apparent effect of the walking condition. This result implies the trajectory on the plane is maintained even if the walking condition is changed.

There are a few studies, particularly those using statistical methods, that imply the existence of a factor in joint movement that is invariant under changing walking conditions: that is, a change in condition is not considered to affect the 
trajectory on the intersegmental plane directly (Courtine and Schieppati 2004; Ivanenko et al. 2007). This supports our result.

\section{Is the tilt of the constraint planes affected?}

From the result of the independent analyses of $z_{1}$ and $z_{2}$, half of the subjects exhibit an effect of velocity on the coordinate plane $z_{1}-z_{2}$, and most of the subjects exhibit an effect of gradient on the plane. However, there are large correlations between the results for different conditions (Table 4). Thus, it was not clear from the independent analyses of $z_{1}$ and $z_{2}$ whether the plane (intersegmental coordination) was variant or invariant.

By embedding the plane in the three-dimensional space, and comparing the normal vectors which represent the tilt of the constraint plane, the plane can be shown to be affected by the walking condition (Figs. 9(i) and (ii)).

The effects on the intersegmental coordination plane of both walking velocity (Ivanenko et al. 2007) and the gradient of the slope (Noble and Prentice 2008) have been discussed previously. Both papers assert that the plane was affected by condition. Our results, even though there are several differences in the derivation of the intersegmental plane, agree with these conclusions.

\section{Is the location of the constraint planes affected?}

The locations of the origins of the intersegmental planes $r_{0}$ change according to velocity and gradient in both orientation $\left\langle r_{0}\right\rangle$ and gain $\left\|r_{0}\right\|$. In particular, $\left\|r_{0}\right\|$, which represents the average of all elevation angles, grows with an increase in velocity or gradient. The actual average postures for the $0[\%], 4[\%], 8[\%]$ and 12[\%] slopes are shown in Fig. 10 which demonstrate the gain of all elevation angles increases as the incline increases.

These results agree with the conventional view that every joint increases its angle when walking on slopes (Leroux et al. 2002; Vogt and Banzer 1999).

\section{Supposed tuning parameters for conditional change}

Based on the above discussion, the whole body movement of human walking forms a cyclic motion independent of the walking condition $\left(\lambda_{1} v_{1}, \lambda_{2} v_{2}\right.$ are constant), while the balance of flexion level among joints (constraint planes 
defined by $z_{1}, z_{2}$ and $r_{0}$ ) is tuned. The result that the loop pattern on the constraint plane is not affected by variance in the environment leads to the interesting outcome that only some selected factors in whole body movement are used as tuning parameters.

The existence of parameters that are robust against changes in velocity and gradient has been implied in papers on the intersegmental coordination of the joints in the lower limbs (Grasso et al. 2000; Bianchi et al. 1998; Noble and Prentice 2008; Ivanenko et al. 2008). However, there was no statistical discussion, and evidence of the invariance of the cycle pattern was not shown. The tilt of pelvis and trunk in response to velocity and gradient escalation has been described in several papers (Murray et al. 1984; Lay et al. 2006; Leroux et al. 2002; Vogt and Banzer 1999). However, a unified analysis, including the changes in both the trunk and lower limbs, has not been previously performed. Therefore, our results that the temporal coordination on the intersegmental plane is statistically robust against changes in the walking condition and that the variation caused by walking condition applies on the level and the balance of joints are new.

\section{The motion of the trunk in comparison to the other joints}

Figure 11 shows the intersegmental coordination for the trunk alone. In DS $z_{2}>\mathrm{Z}_{1}$, while $z_{1}$ and $z_{2}$ are almost the same in SS, regardless of velocity and slope. Furthermore, the variation originating from the condition affects $z_{2}$ in DS the most. It can also be seen that the pattern of responses of $z_{1}$ and $z_{2}$ to an increase in load (faster velocity and larger gradient) is independent of the nature of that load.

As mentioned in the previous section, the contribution of the trunk to the intersegmental coordination is low (Fig. 2), because of the smallness of its motion depicted in Fig. 1(ii). However, when we focus on the mean posture (Fig. 6), the trunk shows high values compared to the differences in motion. In particular, the change in the mean posture (Fig. 8) is at a level that is almost equal with other segments.

Although the motion of trunk is small compared to the others, the contribution of the trunk to intersegmental coordination and the mean posture has 
characteristics that are qualitatively identical and quantitatively similar to those of other links with respect to the tuning level of the mean posture.

\section{The relationship between the motions of DS and SS phases}

In this research, the walking cycles were separated into DS and SS phases and the motions of the two phases were independently analyzed. The reason for this separation is that the dynamic constraint differs between DS and SS, so the recorded motion varies even if the input signal from the central nervous system (CNS) is identical, and this will disturb the identification of the characteristics of motion. However, the separate analyses of DS and SS cause the problem that the properties obtained for the DS and SS phases are unrelated. We solve this problem by embedding the constraint planes of DS and SS into one space as shown in Fig. 3. This embedding also makes possible a quantitative study of the two constraint planes in terms of their normal vectors.

Figure 9(iii) shows that the relative angles between the DS and SS planes are about $50[\mathrm{deg}]$, while the changes in angle due to differences in condition are less than about 15[deg] (Fig. 9(i)).

Therefore, the difference between DS and SS seems essential when considering kinematic properties. Even so, this result does not inevitably indicate that the control signal is independently generated for DS and SS at the level of the CNS. The difference is more probably the effect of the difference in the dynamic constraint, as assumed at the beginning.

\section{Possible problems with the discussion of intersegmental coordination}

In this research, motion tuning is discussed only in terms of the kinematic aspect of the variation, and this has enabled the clear distinction between variant and invariant parameters behind the motion. However, it is more natural to regard kinematic change as a collateral phenomenon of the kinetic tuning of muscle input, because the kinetic elements are more directly connected to the CNS. The 
problem of whether kinematic movement plays a substantial role in control has long been discussed (Borghese et al. 1996; Bianchi et al. 1998; Grasso et al. 1998; Shen and Poppele 1995; Lacquaniti et al. 2002). For example, Lacquaniti et al. compared the variation of COM position and elevation angles of a standing cat in response to weights loaded on its back (Lacquaniti and Maioli 1994) and changing slope (Lacquaniti et al. 2002). They showed that the elevation angles are more robust to changes in condition than $\mathrm{COM}$ position. Based on this result, they conclude that the posture control used kinematics as its control variables (Lacquaniti et al. 2002). Furthermore, human muscle patterns during backward walking differ considerably from those during forward walking. In contrast, the joint angular patterns are a quasi reversal of the patterns in forward walking (Thorstensson 1996; Grasso et al. 1998). This phenomenon also supports the importance of kinematic parameters as the measure of tuning parameters.

It is possible that the intersegmental motion might be irrelevant to neural signaling and control, but is instead constructed by the physical constraints of the joints and environment. Because the same question occurs when considering the intersegmental correlation of the lower limbs, this possibility has been discussed by Ivanenko et al. (2008). They concluded that the plane is not a trivial consequence of the movement by demonstrating that a stoop movement is a higher dimensional correlation and stepping is a lower dimensional correlation. Furthermore, Barliya et al. (2009) analyzed the reason why the correlation plane is formed by the three elevation angles of the lower limbs. They derived the condition for the planar trajectory that the frequencies of the motion for the three elevation angles are equal, a condition which is roughly satisfied in human walking.

\section{Relationship with the control in muscles and higher center}

It is well known that the correlated activation occurring among joints is also observed in the case of muscles. For example, the activation patterns recorded in 25 muscles during walking can be constructed from five independent patterns (Olree and Vaughan 1995; Ivanenko et al. 2004). Similar movements, such as obstacle avoidance and ball kicking, can be built by adding one other component (Ivanenko et al. 2005). Moreover, signals corresponding to the intersegmental 
correlation are observed in the dorsal spinocerebellar tract neurons of cats (Poppele et al. 2002).

Because the characteristics of the correlation between the muscles and neurons include information about the control signals, and the characteristics of the correlation in the kinematics include information about the resulting action, it will be essential to understand the relationship between the two correlations for further investigation of the control signal and control procedures. The current research presents a characteristic feature for the tuning of posture in response to environmental changes. This will be helpful both when connecting the kinematics and muscles via a common feature and when finally locating the adaptation in the control system.

\section{Conclusions}

We have investigated the adaptive changes of the motion pattern during walking for different inclines and walking velocities. We focused on the intersegmental coordination of the whole body, particularly emphasizing the correlation of the trunk and lower limbs. Our main results are (1) the whole body movement can be decomposed into two intersegmental coordinations, two temporal coordinations and the mean posture; (2) the movement can be described as a closed loop on twodimensional constraint planes in joint space; (3) under different conditions, the tilt and the position of the constraint planes change; and (4) the temporal coordination of walking is robust against changes in walking conditions. From these results, we conclude that humans tune only some selected properties in response to walking conditions. This enables a reduction in the number of control parameters and a simple strategy for adaptation.

\section{Acknowledgement}

This paper was supported in part by a Grant-in-Aid for Creative Scientific Research (No. 19GS0208) from the Ministry of Education, Culture, Sports, Science, and Technology of Japan. 


\section{Appendix: Description of walking motion on joint spaces}

In order to describe the motion of DS and SS in the same space, the time series of the motion $R(t)$ and the constraint planes $\left(z_{1}^{\mathrm{DS}}, z_{2}^{\mathrm{DS}}\right),\left(z_{1}^{\mathrm{SS}}, z_{2}^{\mathrm{SS}}\right)$ are embedded in the $\left(\tilde{z}_{1}, \tilde{z}_{2}, \tilde{z}_{3}\right)$ space built by the movement when DS and SS phases are considered together. The posture at time $t: R(t)$ in this space is

$$
\widetilde{R}_{i}(t)=\left(R(t)-\widetilde{R}_{0}\right) \cdot \tilde{z}_{i}, \quad(i=1 \cdots 3)
$$

where $\widetilde{R}_{0}$ is the mean posture over both DS and SS phases.

The spaces $\tilde{z}^{\text {DS }}$ and $\tilde{z}^{\text {SS }}$ are embedded with origins $\tilde{z}^{\text {0DS }}$ and $\tilde{z}^{\text {oss }}$ determined from the mean postures $R_{0}^{\mathrm{DS}}$ and $R_{0}^{\mathrm{SS}}$ by

$$
\left\{\begin{array}{l}
\tilde{z}_{i}^{0 \mathrm{DS}}=\left(R_{0}^{\mathrm{DS}}-\widetilde{R}_{0}\right) \cdot \tilde{z}_{i} \\
\tilde{z}_{i}^{0 \mathrm{SS}}=\left(R_{0}^{\mathrm{SS}}-\widetilde{R}_{0}\right) \cdot \tilde{z}_{i}
\end{array}\right.
$$

The axes of the intersegmental coordination $\left(\tilde{z}_{i}^{j D S}, \tilde{z}_{i}^{j S S}\right)$ are determined from $z_{j}^{\mathrm{DS}}, z_{j}^{\mathrm{SS}}(j=1,2)$ when DS and SS are considered separately:

$$
\left\{\begin{array}{l}
\tilde{z}_{i}^{j D S}=z_{j}^{D S} \cdot \tilde{z}_{i} \\
\tilde{z}_{i}^{j S S}=z_{j}^{S S} \cdot \tilde{z}_{i}
\end{array} \quad(i=1 \cdots 3, j=1 \cdots 2) .\right.
$$




\section{References}

Alexandrov A, Frolov A, Massion J (1998) Axial synergies during human upper trunk bending. Exp Brain Res 118:210-220.

Anders C, Wagner H, Puta C, Grassme R, Petrovitch A, Scholle H-C (2007) Trunk muscle activating patterns during walking at different speeds. J Electromyography and Kinesiology 17:245-252.

Barliya A, Omlor L, Giese MA, Flash T (2009) An analytical formulation of the law of intersegmental coordination during human locomotion. Exp Brain Res 193:371-385.

Berret B, Bonnetblanc F, Papaxanthis C, Pozzo T (2009) Modular control of pointing beyond arm's length. J Neurosci 29(1):191-205.

Bianchi L, Angelini D, Orani GP, Lacquaniti F (1998) Kinematic coordination in human gait: relation to mechanical energy cost. J Neurophysiol 79: 2155-2170.

Borghese NA, Bianchi L, Lacquaniti F (1996) Kinematic determinants of human locomotion. J Physiol 494(3):863-879.

Courtine G, Schieppati M (2004) Tuning of a basic coordination pattern constructs straight-ahead and curved walking in humans. J Neurophysiol 91: 1524-1535.

Cromwell RL (2003) Movement strategies for head stabilization during incline walking. Gait and Posture 17: 246-253.

Crosbie J, Vachalathiti R (1997) Synchrony of pelvic and hip joint motion during walking. Gait and Posture 6: 237-248.

Frigo C, Carabalona R, Mura MD, Negrini S (2003) The upper body segmental movements during walking by young females. Clin Biomechanics 18:419-425.

Grasso R, Bianchi L, Lacquaniti F (1998) Motor patterns for human gait: backward versus forward Locomotion. J Neurophysiol 80:1868-1885.

Grasso R, Zago M, Lacquaniti F (2000) Interactions between posture and locomotion: motor patterns in humans walking with bent posture versus erect posture. J Neurophysiol 83;288-300.

Iida H, Yamamuro T (1987) Kinetic analysis of the center of gravity of the human body in normal and pathological gaits. J Biomechanics 20(10):987-995.

Ivanenko YP, Poppele RE, Lacquaniti F (2004) Five basic muscle activation patterns account for muscle activity during human locomotion. J Physiol 556(1):267-282.

Ivanenko YP, Cappelline G, Dominici N,Poppele RE, Lacquaniti F (2005) Coordination of locomotion with voluntary movements in human. J Neurosci 25(31):7238-7253.

Ivanenko YP, Cappellini G, Dominici N, Poppele RE, Lacquaniti F (2007) Modular control of limb movements during human locomotion. J Neurosci 27(41):111490-11161.

Ivanenko YP, d'Avella A, Poppele RE, Lacquaniti F (2008) On the origin of planar covariation of elevation angles during human locomotion. J Neurophysiol 99: 1890-1898.

Lacquaniti F, Taillanter ML, Lopiano L, Maioli C (1990) The control of limb geometry in cat posture. J Physiol 426:177-192. 
Lacquaniti F, Maioli C (1994) Independent control of limb position and contact forces in cat posture. J Neurophysiol 72:1476-1495.

Lacquaniti F, Ivanenko YP, Zago M (2002) Kinematic control of walking. Archives Italiennes de Biologie 140:263-272.

Lay AN, Hass CJ, Gregor RJ (2006) The effects of sloped surfaces on locomotion: A kinematic and kinetic analysis. (2006) J Biomechanics 39:1621-1628.

Lay AN, Hass CJ, Nichols TR, Gregor RJ (2007) The effects of sloped surfaces on locomotion: An electromyographic analysis. J Biomechanics 40: 1276-1285.

Leroux A, Fung J, Barbeau H (2002) Postural adaptation to walking on inclined surfaces: I. Normal strategies. Gait and Posture 15:64-74.

Mah CD, Hulliger M, Lee RG, O'Callaghan I (1994) Quantitative analysis of human movement synergies: constructive pattern analysis for gait. J Motor Behavior 26:83-102.

Martin SV, Martin N, Massion J (1999) Kinematic synergies and equilibrium control during trunk movement under loaded and unloaded conditions. Exp Brain Res 128: 517-526.

Martin SV, Martin N, Massion J (2000) Kinematic synergy adaptation to microgravity during forward trunk movement. J Neurophysiol 83:453-464.

Milner M, Quanbury AO (1970) Facets of control in human walking. Nature 227:734-735.

Murray MP, Drought AB, Kory RC (1964) Walking patterns of normal men. J Bone Joint Surg Am 46: 335-360.

Murray MP, Mollinger LA, Gardner GM, Sepic SB (1984) Kinematic and EMG patterns during slow, free, and fast walking. J Ortho Res 2:272-280.

Noble JW, Prentice SD (2008) Intersegmental coordination while walking up inclined surfaces: age and ramp angle effects. Exp Brain Res 189: 249-255.

Olree KS, Vaughan CL (1995) Fundamental patterns of bilateral muscle activity in human Locomotion. Biological Cybernetics 73:409-414.

Poppele RE, Bosco G, Rankin AM (2002) Independent representations of limb axis length and orientation in spinocerebellar response components. J Neurophysiol 87:409-422.

St-Onge N, Feldman AG (2003) Interjoint coordination in lower limbs during different movements in humans. Exp Brain Res 148:139-149.

Sartor C, Alderink G, Greenwald H, Elders L (1999) Critical kinematic events occurring in the trunk during walking. Human Mov Sci 18(5): 669-679.

Shen L, Poppele R (1995) Kinematic analysis of cat hindlimb stepping. J Neurophysiol 74: 22662280.

Stoquart G, Detrembleur C, Lejeune T (2008) Effect of speed on kinematic, kinetic, electromyographic and energetic reference values during treadmill walking. Clin Neurophysiol 38:105116.

Thomas JS, Corcos DM, Hasan Z (2005) Kinematic and kinetic constraints on arm, trunk, and leg segments in target-reaching movements. J Neurophysiol 93:352-364.

Thorstensson A (1986) How is the normal locomotor program modified to produce backward walking? Exp Brain Res 61:664-668. 
Vogt L, Banzer W (1999) Measurement of lumbar spine kinematics in incline treadmill walking. Gait and Posture 9: 18-23.

Winter DA (1987) The biomechanics and motor control of human gait. University of Waterloo Press.

Yamazaki M, Sasaki T, Tsuzuki S, Torii M (1984) Stereotyped pattern of lower limb movement during level and grade walking on treadmill. Annals of Physiological Anthropol 3(4):291-296. 


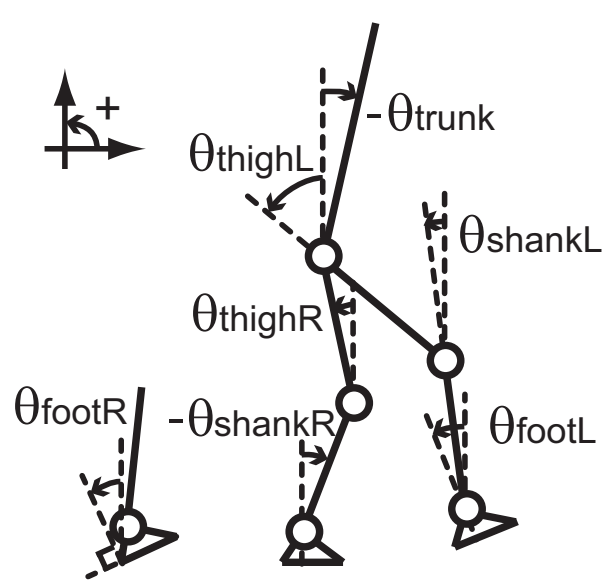

(i) Definitions of elevation angles

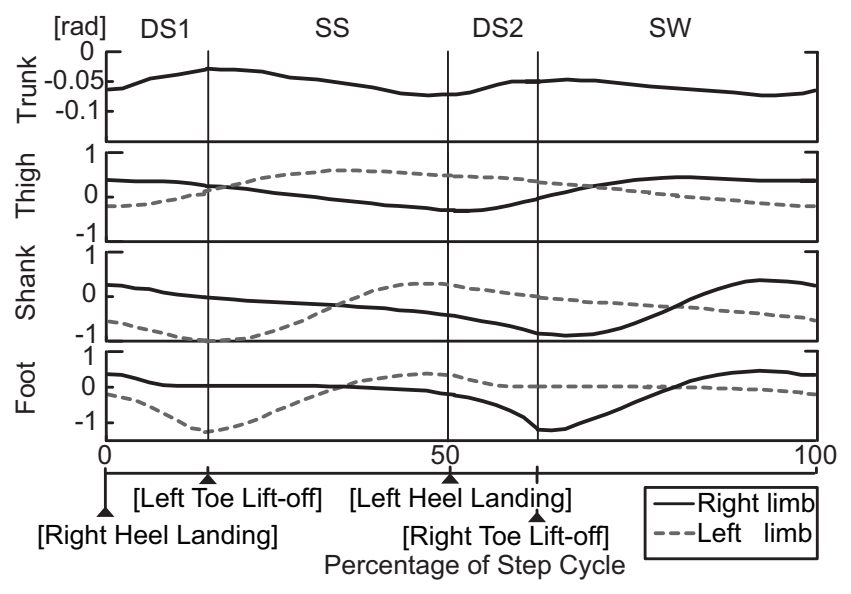

(ii) Time series of 7 elevation angles (subject: $\mathrm{HO}, 3[\mathrm{~km} / \mathrm{h}]$ )

Fig. 1 (i) Definition of elevation angles, that is, the angle from a vertical line, and (ii) calculated time series of the elevation angles. The calculated angles are separated into double support phases (DS1, DS2) and single support phases (SS, SW) according to the timing of heel landing and toe lift-off. In (ii) segments of the right lower limbs are depicted by solid lines, and left lower limbs are depicted by dotted lines. 


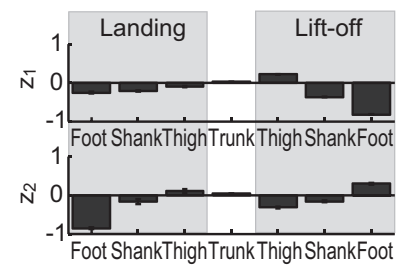

(a) Double support phase

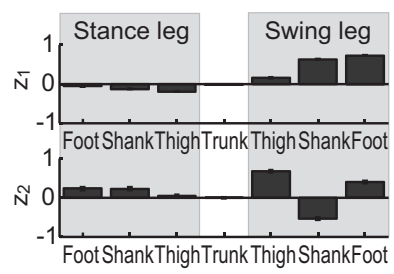

(b) Single support phase

(i) Intersegmental coordination $(z i)$
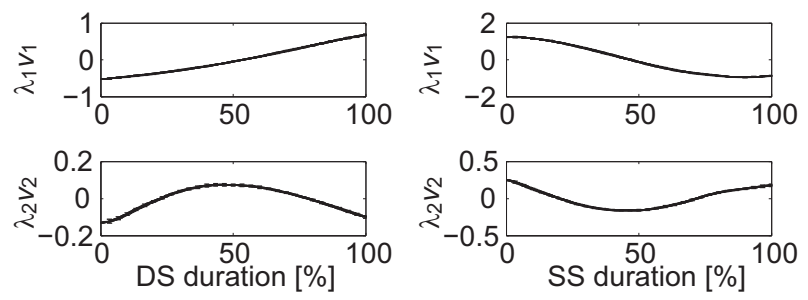

(a) Double support phase (b) Single support phase

(ii) Temporal coordination $\left(\lambda_{i} v_{i}\right)$

Fig. 2 Intersegmental and temporal coordination obtained by singular value decomposition (SVD). By performing SVD on the recorded time series of motion, joint groups and temporal groups with high correlations are extracted. (i) The intersegmental coordination shows the groups of segments which activate simultaneously, and (ii) the activation pattern is depicted as a temporal coordination. In (i) landing and liftoff are used to indicate the right and left leg in DS1, while stance and swing leg indicate the right and left leg in SS. The patterns shown are those of subject HO walking on level ground at $3[\mathrm{~km} / \mathrm{h}]$. The charts are composed from the average and the standard deviation for 14 cycles calculated from motion under the same condition. 


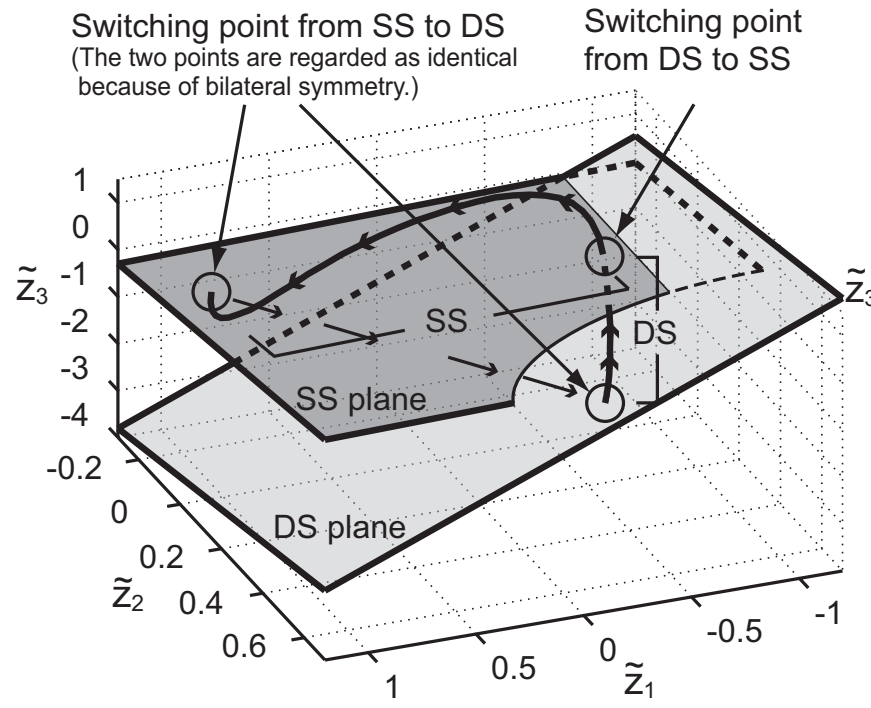

3 dim. sub-space spanned by interjoint coordination of cycle in $7 \mathrm{dim}$. joint space

(i) Constraint planes and posture calculated for level ground

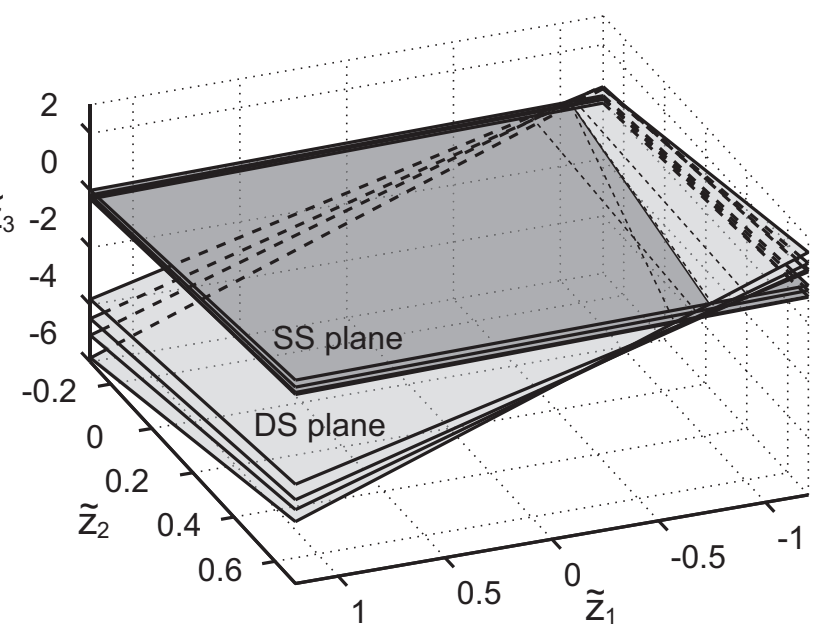

3 dim. sub-space spanned by interjoint coordination of cycle in $7 \mathrm{dim}$. joint space

(ii) Constraint planes calculated for various slope conditions

Fig. 3(i) The whole body movement can be described in a three-dimensional sub-space of the seven-dimensional joint space. The axes are defined by the three intersegmental coordinations calculated from the whole joint movement of DS1 and SS, and the path indicated by the arrows shows the time series of the posture $R(t)$. The dashed lines indicate that these lines lie beneath one or more overlying planes. Two planes can be built in the three-dimensional space, expressing the movement in DS and SS phases. These planes are defined by axes $z_{1}(\theta)$ and $z_{2}(\theta)$ with $r_{0}$ as the origin. (ii) The planes induced by motion under different slope conditions $(0,4,8,12[\%])$. The difference in the tilt of the planes for each phase are small compared to the angle between the DS and SS planes. The figure is constructed from the data of subject KU. 

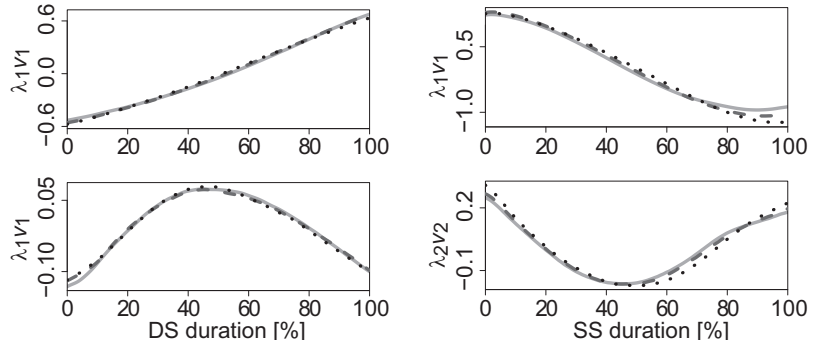

(a) Double support phase
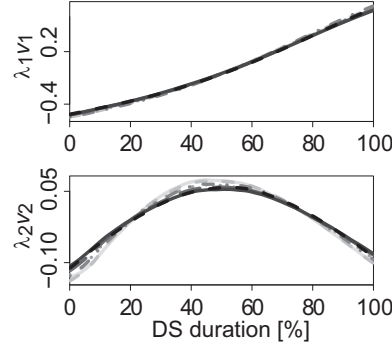

(a) Double support phase
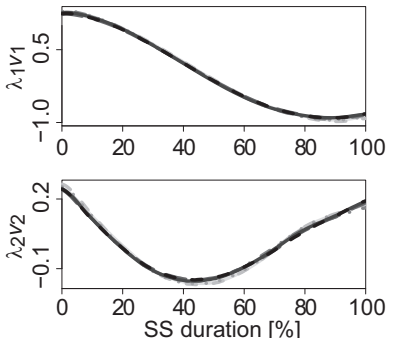

(b) Single support phase

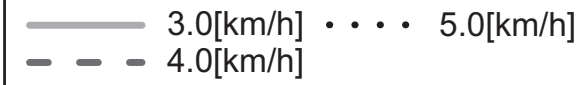

(i) Temporal coordinations obtained at different velocities

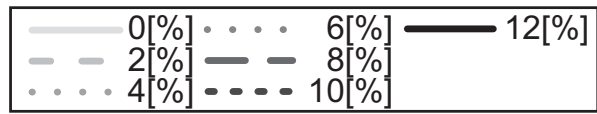

(ii) Temporal coordinations obtained on different inclines

Fig. 4 Temporal coordinations computed for different velocities and slope angles. The pattern shown is the average of many patterns obtained per step cycle under the same walking condition. The walking conditions are (i) velocities of $3,4,5[\mathrm{~km} / \mathrm{h}]$ and (ii) inclines of $0-12[\%]$. Most of the patterns are overlain by others. The data is from subject HO. The charts show the average of data obtained under the same walking conditions. 


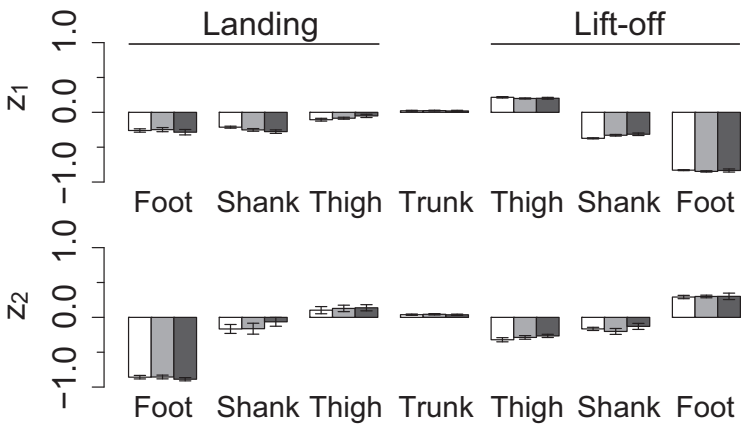

(a) Double support phase

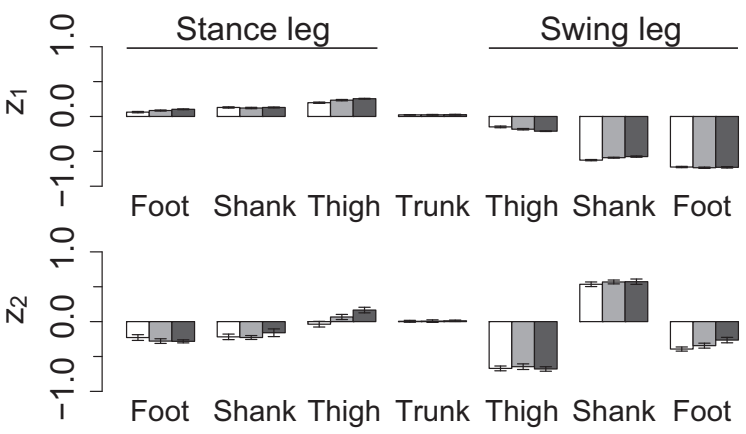

(b) Single support phase

\begin{tabular}{|l|l|}
\hline $3.0[\mathrm{~km} / \mathrm{h}]$ & $\square .0[\mathrm{~km} / \mathrm{h}]$ \\
$\square$ & \\
$\square$ & \\
&
\end{tabular}

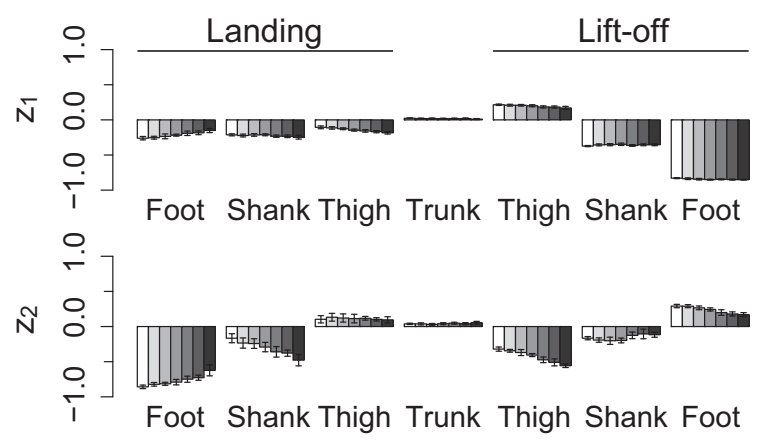

(a) Double support phase

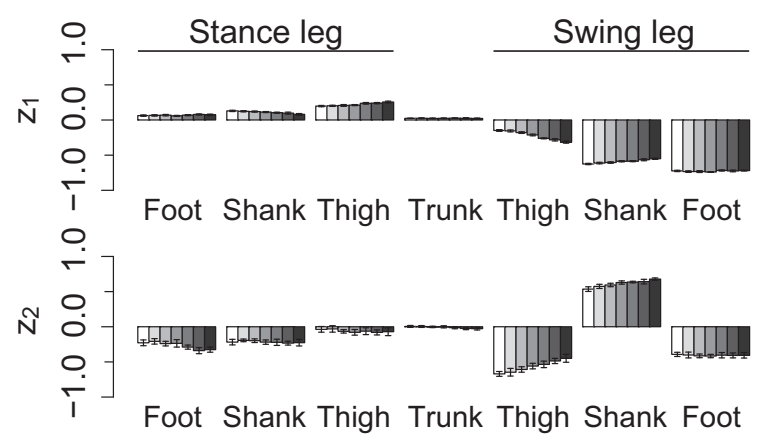

(b) Single support phase

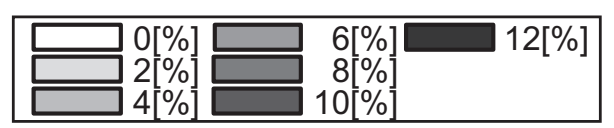

(i) Intersegmental coordinations obtained at different velocities (ii) Intersegmental coordinations obtained on different inclines

Fig. 5 Intersegmental coordinations computed from motion data recorded for (i) different velocities and (ii) different inclines. The patterns remain similar even when the walking conditions change, but it is possible to find a few clear effects of condition: for example, in (ii) $z_{2}$ gradually changes with an increase in the slope angle. The data is from subject HO. The charts show the average and the standard deviation of data obtained under the same walking conditions. 


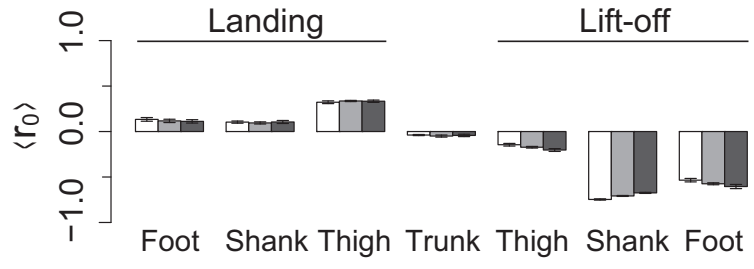

(a) Double support phase

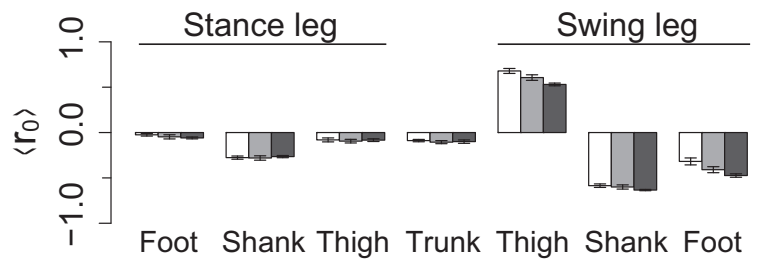

(b) Single support phase

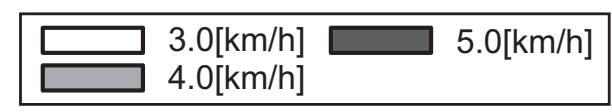

(i) Normalized mean posture at different velocities

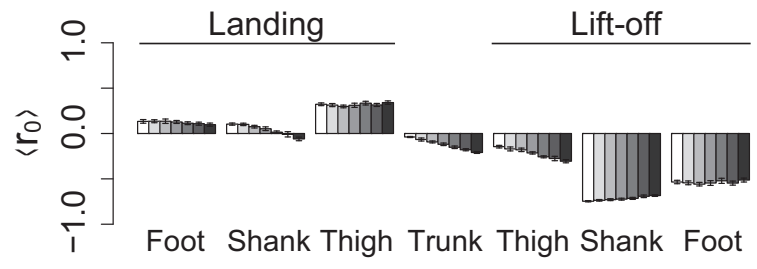

(a) Double support phase

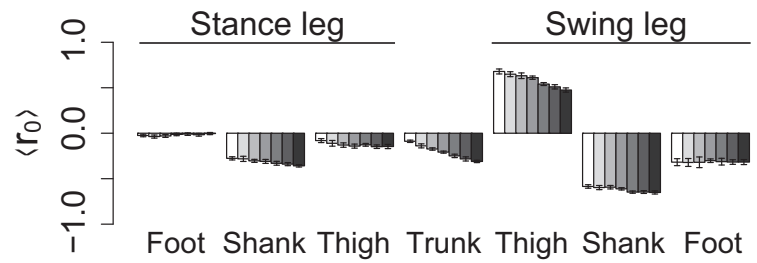

(b) Single support phase

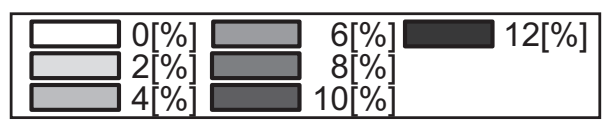

(ii) Normalized mean posture on different inclines

Fig. 6 Intersegmental coordination of mean posture $\left(<r_{0}>\right)$. By normalizing the mean posture, the intersegmental coordination of the posture can be derived. Differences due to walking condition can be seen, particularly in the trunk and thigh. The significance of the difference is confirmed using 2-way ANOVA with factors (joints $\times$ conditions). The data is for subject HO. The charts show the average and the standard deviation of data obtained under the same walking conditions. 


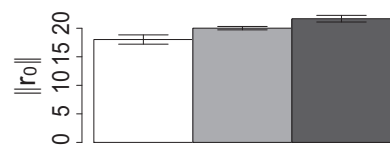

(a) Double support phase

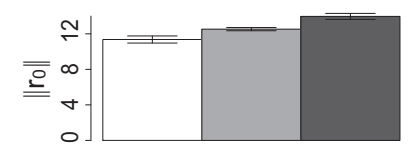

(b) Single support phase

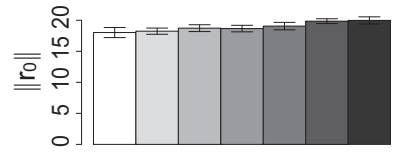

(a) Double support phase

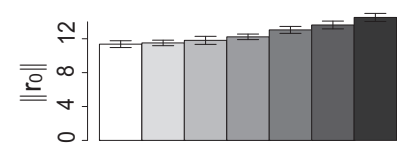

(b) Single support phase

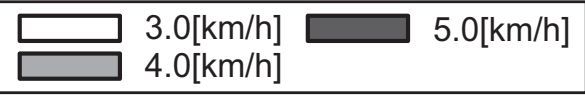

(i) Gain of mean posture at different velocities

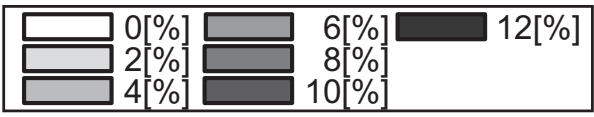

(ii) Gain of mean posture on different inclines

Fig. 7 Gain of mean posture $\left(\left\|r_{0}\right\|\right)$ recorded for various velocities and gradients. The gain apparently grows as the velocity or incline increases, and this trend is confirmed by a 1-way ANOVA. The data is from subject HO. The charts show the average and the standard deviation of data obtained under the same walking conditions. 


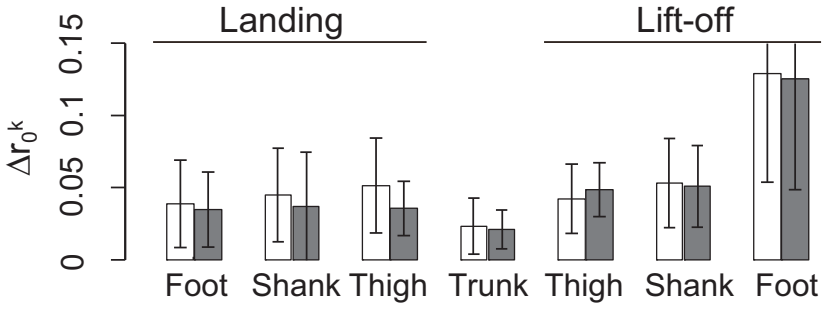

(a) Double support phase

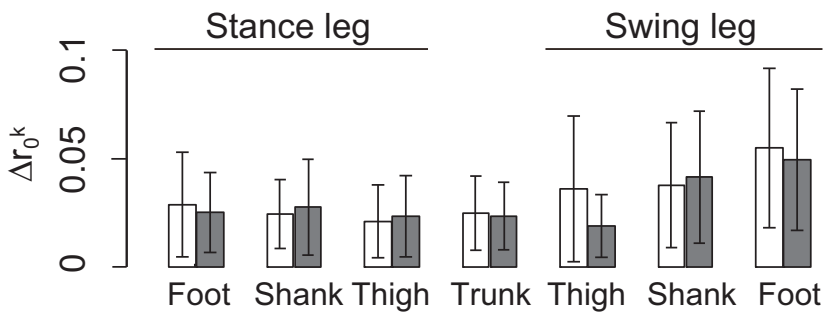

(b) Single support phase

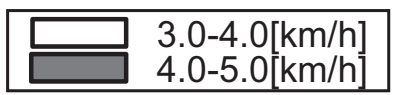

(i) Variation at different velocities

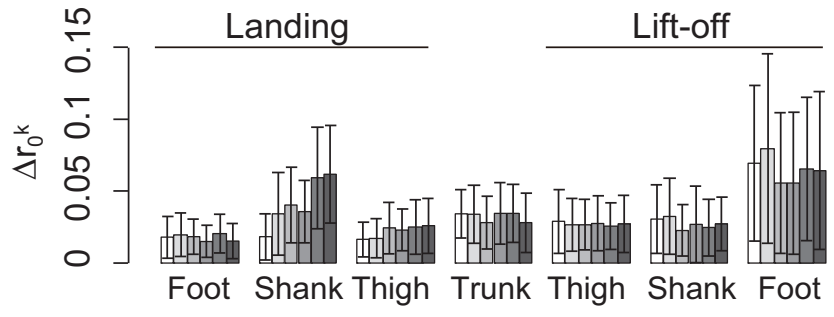

(a) Double support phase

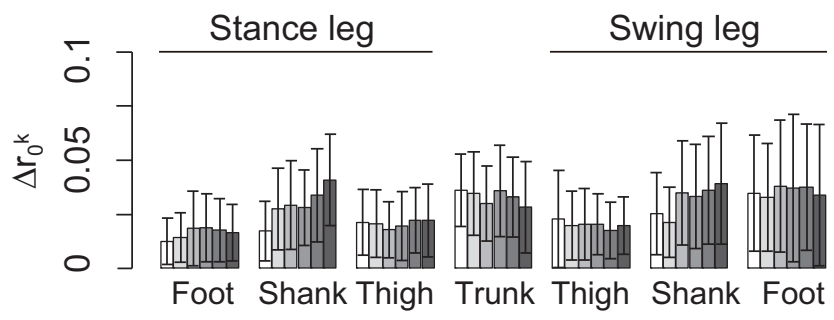

(b) Single support phase

\begin{tabular}{|c|c|c|}
\hline $0-2[\%]$ & $4-6[\%]$ & 8-10[\%] \\
\hline $2-4[\%]$ & $6-8[\%]$ & $10-12[\%]$ \\
\hline
\end{tabular}

(ii) Variation on different slopes

Fig. 8 Change in the elevation angles of mean posture. Bars represent the difference in the elevation angles of each joint calculated for two conditions. The values for all trials and all subjects are combined and the average and standard deviation are displayed. The changes are approximately the same for every joint, while the highest variability occurs for the foot of the lift-off and swing legs. 

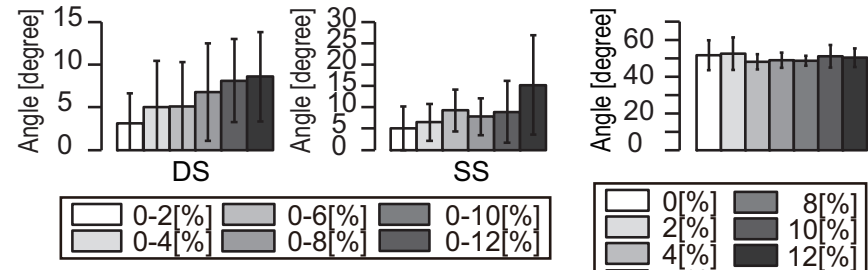

(i) Relative angles among different slopes

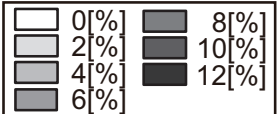

(iii) Relative angles between
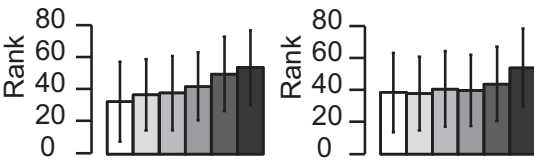

DS and SS planes

DS

SS

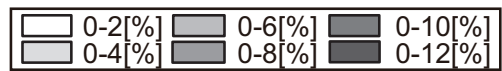

(ii) Rank of relative angles among different slopes

Fig. 9 The relative angles of normal vectors of constraint planes. In (i) and (ii) the constraint planes of level gound and each gradient are compared. The angles is described by (i) the absolute value and (ii) the rank of the value in that subject in order to remove the difference among subjects. (iii) displays the relative angles between DS and SS. Values for all subjects and all trials are summarized and displayed as average values and standard deviations. 


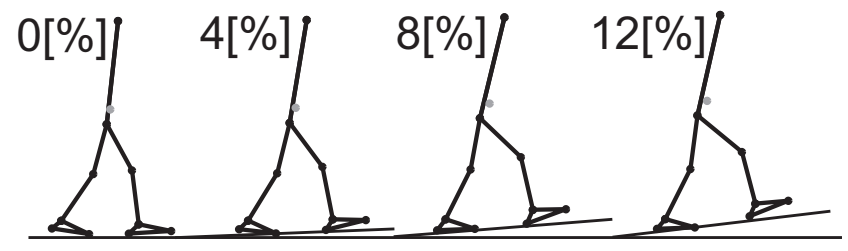

(i) Double support phase

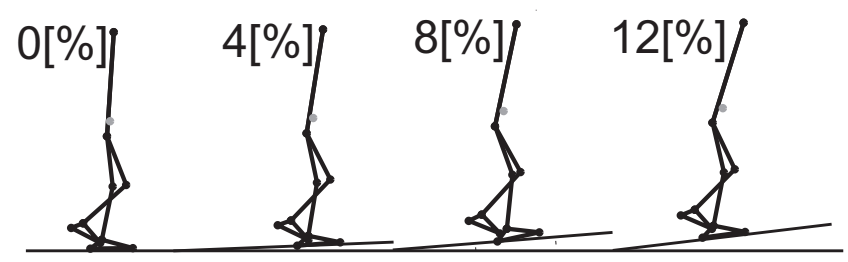

(ii) Single support phase

Fig. 10 Mean posture of walking on $0[\%]$ to $12[\%]$ inclines in the double and single support phases. The amplitudes of all elevation angles increase and the bending level of the body angle increases as a result. Displayed posture is generated from the walking of subject $\mathrm{HO}$ at $3.0[\mathrm{~km} / \mathrm{h}]$. 

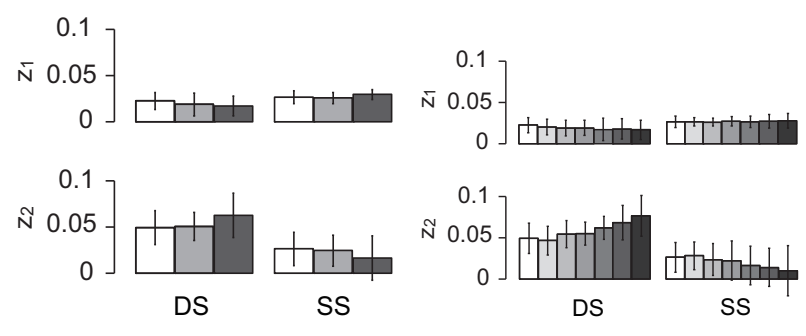

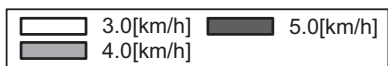

(i) Different velocities

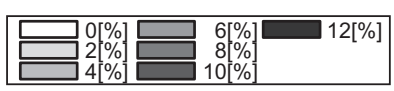

(ii) Different slopes

Fig. 11 Intersegmental coordination of the trunk under various conditions. The values of all trials of all subjects are combined and the average and standard deviation are displayed. This is done in order to absorb the variance caused by the minuteness of the intersegmental coordination of the trunk. 
Table 1 Singular value $\lambda_{i}$ and cumulative proportion for 10 subjects on level ground. Listed values are the average of 14 steps under the same conditions and values in brackets are the standard deviations.

(i) SVD results for the DS and SS phases

\begin{tabular}{|c|c|c|c|c|c|c|c|c|}
\hline \multirow[b]{3}{*}{ Subject } & \multicolumn{4}{|c|}{ DS } & \multicolumn{4}{|c|}{ SS } \\
\hline & \multicolumn{2}{|c|}{ Singular Value } & \multicolumn{2}{|c|}{$\begin{array}{l}\text { Cumulati- } \\
\text { ve Prop. }\end{array}$} & \multicolumn{2}{|c|}{ Singular Value } & \multicolumn{2}{|c|}{$\begin{array}{c}\text { Cumulati- } \\
\text { ve Prop. }\end{array}$} \\
\hline & $\lambda_{1}$ & $\lambda_{2}$ & 1 & 2 & $\lambda 1$ & $\lambda_{2}$ & 1 & 2 \\
\hline $\mathrm{HO}$ & $6.33(0.2)$ & $1.08(0.1)$ & 0.97 & 1.00 & $13.6(0.3)$ & $2.16(0.1)$ & 0.97 & 1.00 \\
\hline $\mathrm{AD}$ & $5.12(0.4)$ & $0.52(0.1)$ & 0.99 & 1.00 & $11.9(0.4)$ & $2.01(0.1)$ & 0.97 & 1.00 \\
\hline MA & $5.20(0.4)$ & $0.61(0.1)$ & 0.99 & 1.00 & $12.8(0.5)$ & $1.20(0.1)$ & 0.99 & 1.00 \\
\hline $\mathrm{KI}$ & $4.66(0.8)$ & $0.58(0.1)$ & 0.98 & 1.00 & $12.1(0.4)$ & $1.65(0.1)$ & 0.98 & 1.00 \\
\hline $\mathrm{KO}$ & $5.79(0.3)$ & $0.98(0.1)$ & 0.97 & 1.00 & $13.1(0.4)$ & $1.69(0.1)$ & 0.98 & 1.00 \\
\hline MO & $3.05(0.5)$ & $0.47(0.1)$ & 0.98 & 1.00 & $11.6(0.3)$ & $1.98(0.1)$ & 0.97 & 0.99 \\
\hline NA & $5.61(0.7)$ & $1.08(0.2)$ & 0.96 & 1.00 & $12.8(0.9)$ & $1.83(0.2)$ & 0.98 & 1.00 \\
\hline MI & $3.19(2.1)$ & $0.46(0.4)$ & 0.98 & 1.00 & $11.9(0.5)$ & $2.42(1.0)$ & 0.95 & 0.99 \\
\hline KU & $4.09(0.4)$ & $0.59(0.1)$ & 0.98 & 1.00 & $12.1(0.4)$ & $1.79(0.2)$ & 0.97 & 0.99 \\
\hline AN & $3.94(0.8)$ & $0.60(0.2)$ & 0.98 & 1.00 & $11.1(0.2)$ & $1.71(0.3)$ & 0.97 & 1.00 \\
\hline
\end{tabular}

(ii) SVD results for DS and SS phases combined DS-SS

\begin{tabular}{ccccccc}
\hline \multicolumn{3}{c}{ Singular Value } & & \multicolumn{3}{c}{$\begin{array}{c}\text { Cumulative } \\
\text { Proportion }\end{array}$} \\
\hline$\lambda_{1}$ & $\lambda 2$ & $\lambda 3$ & & 1 & 2 & 3 \\
\cline { 1 - 3 } \cline { 5 - 6 } $13.2(0.3)$ & $4.92(0.2)$ & $2.01(0.1)$ & & 0.86 & 0.98 & 1.00 \\
$11.3(0.4)$ & $4.62(0.2)$ & $1.46(0.1)$ & & 0.84 & 0.98 & 1.00 \\
$12.8(0.6)$ & $3.76(0.3)$ & $1.22(0.1)$ & & 0.91 & 0.99 & 1.00 \\
$11.6(0.5)$ & $4.42(0.3)$ & $1.38(0.1)$ & & 0.86 & 0.99 & 1.00 \\
$12.5(0.5)$ & $4.80(0.4)$ & $1.60(0.1)$ & & 0.86 & 0.98 & 1.00 \\
$11.3(0.4)$ & $3.69(0.3)$ & $1.52(0.1)$ & & 0.89 & 0.98 & 1.00 \\
$12.1(1.0)$ & $5.16(0.5)$ & $1.46(0.2)$ & & 0.83 & 0.99 & 1.00 \\
$11.5(0.5)$ & $4.32(0.2)$ & $1.39(0.1)$ & & 0.86 & 0.99 & 1.00 \\
$11.4(0.3)$ & $4.27(0.2)$ & $1.49(0.2)$ & & 0.86 & 0.98 & 1.00 \\
$10.8(0.2)$ & $4.03(0.2)$ & $1.26(0.1)$ & 0.87 & 0.99 & 1.00 \\
\hline
\end{tabular}


Table 2 Results of the Kruskal-Wallis rank tests for differences in the average pattern depicted in Fig. 4. Left-hand table shows results for changes in velocity; right-hand table shows results for changing incline. There is no clear effect of walking condition even at the weaker $5 \%$ level of significance.

(i) P-values for differences due to change in velocity.

\begin{tabular}{cccccc} 
& \multicolumn{2}{c}{ DS } & & \multicolumn{2}{c}{ SS } \\
\cline { 2 - 3 } \cline { 5 - 6 } Subject & $\lambda 1 \mathrm{~V} 1$ & $\lambda 2 \mathrm{~V} 2$ & & $\lambda 1 \mathrm{~V} 1$ & $\lambda 2 \mathrm{~V} 2$ \\
\hline HO & 0.996 & 0.821 & & 0.870 & 0.783 \\
AD & 0.996 & 1.000 & & 0.489 & 0.872 \\
MA & 0.996 & 0.759 & & 0.985 & 0.625 \\
KI & 0.980 & 0.928 & & 0.141 & 0.605 \\
KO & 0.977 & 0.983 & & 0.431 & 0.793 \\
MO & 0.988 & 0.997 & & 0.931 & 0.936 \\
NA & 0.839 & 0.766 & & 0.073 & 0.564 \\
MI & 0.991 & 0.883 & & 0.082 & 0.186 \\
KU & 0.999 & 0.645 & & 0.903 & 0.955 \\
AN & 0.980 & 0.994 & 0.199 & 0.879
\end{tabular}

(ii) $\mathrm{P}$-values for differences due to change in incline.

\begin{tabular}{cccccc} 
& \multicolumn{2}{c}{ DS } & & \multicolumn{2}{c}{ SS } \\
\cline { 2 - 3 } \cline { 5 - 6 } Subject & $\lambda 1 \mathrm{~V} 1$ & $\lambda 2 \mathrm{~V} 2$ & & $\lambda 1 \mathrm{~V} 1$ & $\lambda 2 \mathrm{~V} 2$ \\
\hline HO & 1.000 & 0.962 & & 0.346 & 0.980 \\
AD & 1.000 & 0.997 & & 0.804 & 0.971 \\
MA & 1.000 & 0.774 & & 0.400 & 0.986 \\
KI & 0.942 & 0.982 & & 0.208 & 0.910 \\
KO & 1.000 & 0.979 & & 0.087 & 0.963 \\
MO & 1.000 & 0.832 & & 0.733 & 0.973 \\
NA & 0.999 & 0.998 & & 0.667 & 0.993 \\
MI & 0.995 & 0.912 & & 0.360 & 0.175 \\
KU & 1.000 & 0.916 & & 1.000 & 0.999 \\
AN & 0.994 & 0.959 & 0.757 & 0.797
\end{tabular}


Table 3 Results of 2-way ANOVA to determine the effects of walking condition using the time-discretised pattern. The average and the standard deviation are calculated in every 10\% segment of the support phase, and the difference is tested using 2-way ANOVA with factors (time $\times$ walking condition (velocity or gradient)). The listed values are the p-values for factors of walking condition. There is no effect of walking condition for more than $80 \%$ of subjects.

(i) P-values for differences due to change in velocity

\begin{tabular}{|c|c|c|c|c|}
\hline \multirow[b]{2}{*}{ Subject } & \multicolumn{2}{|c|}{ DS } & \multicolumn{2}{|c|}{ SS } \\
\hline & $\lambda_{1} \mathrm{~V}_{1}$ & $\lambda_{2} \mathrm{~V}_{2}$ & $\lambda_{1} \mathrm{~V}_{1}$ & $\lambda_{2} \mathrm{~V}_{2}$ \\
\hline $\mathrm{HO}$ & 0.333 & 0.682 & 0.147 & 0.041 \\
\hline $\mathrm{AD}$ & 0.616 & 0.262 & 0.918 & 0.230 \\
\hline MA & 0.888 & 0.789 & 0.498 & 0.242 \\
\hline KI & 0.817 & 0.506 & 0.908 & 0.334 \\
\hline $\mathrm{KO}$ & 0.710 & 0.616 & 0.229 & 0.092 \\
\hline MO & 1.000 & 0.702 & 0.742 & 0.495 \\
\hline NA & 0.935 & $(<0.05)$ & 0.403 & 0.340 \\
\hline MI & 0.992 & 0.556 & 0.631 & 0.896 \\
\hline $\mathrm{KU}$ & $(<0.05)$ & 0.451 & 0.220 & 0.275 \\
\hline AN & 0.933 & 0.487 & 0.938 & 0.268 \\
\hline
\end{tabular}

(ii) P-values for differences due to change in incline

\begin{tabular}{cccccc} 
& \multicolumn{2}{c}{$\mathrm{DS}$} & & \multicolumn{2}{c}{$\mathrm{SS}$} \\
\cline { 2 - 3 } \cline { 5 - 6 } Subject & $\lambda 1 \mathrm{~V}_{1}$ & $\lambda_{2} \mathrm{~V}_{2}$ & & $\lambda_{1} \mathrm{~V}_{1}$ & $\lambda_{2} \mathrm{~V}_{2}$ \\
\hline HO & 0.983 & 0.051 & & 0.657 & 0.843 \\
$\mathrm{AD}$ & 1.000 & 0.317 & & 0.353 & $(<0.01)$ \\
MA & 0.977 & 0.174 & & 0.152 & 0.658 \\
KI & 0.991 & 0.445 & & 0.325 & 0.878 \\
KO & 0.996 & $(<0.01)$ & & 0.204 & 0.730 \\
MO & 1.000 & 0.474 & & $(<0.01)$ & 0.724 \\
NA & 0.956 & $(<0.05)$ & 0.836 & $(<0.05)$ \\
MI & 1.000 & 0.083 & & $(<0.01)$ & 0.621 \\
KU & 0.523 & 0.978 & 0.090 & 0.692 \\
AN & 0.999 & 0.353 & 0.076 & 0.629
\end{tabular}


Table 4 Correlation coefficient of intersegmental coordination for different velocities and inclines. Every pair has a high correlation coefficient, which indicates high similarity between different patterns.

(i) Correlation coeff. at different velocities

\begin{tabular}{lccc} 
& & DS & SS \\
\hline $3.0-4.0[\mathrm{~km} / \mathrm{h}]$ & $\mathrm{Z}_{1}$ & 0.994 & 0.999 \\
& $\mathrm{Z}_{2}$ & 0.975 & 0.978 \\
\hline $4.0-5.0[\mathrm{~km} / \mathrm{h}]$ & $\mathrm{Z}_{1}$ & 0.997 & 0.999 \\
& $\mathrm{Z}_{2}$ & 0.983 & 0.991 \\
\hline $3.0-5.0[\mathrm{~km} / \mathrm{h}]$ & $\mathrm{Z}_{1}$ & 0.983 & 0.996 \\
& $\mathrm{Z}_{2}$ & 0.940 & 0.959 \\
\hline
\end{tabular}

(ii) Correlation coeff. on different inclines

\begin{tabular}{llcc} 
& & DS & SS \\
\hline Level-2[\%] & $\mathrm{Z}_{1}$ & 0.999 & 1.000 \\
& $\mathrm{Z} 2$ & 0.991 & 0.988 \\
\hline 4[\%] -6[\%] & $\mathrm{Z} 1$ & 1.000 & 0.999 \\
& $\mathrm{Z} 2$ & 0.996 & 0.986 \\
\hline 8[\%] -10[\%] & $\mathrm{Z} 1$ & 0.999 & 0.999 \\
& $\mathrm{Z} 2$ & 0.995 & 0.978 \\
\hline Level-12[\%] & $\mathrm{Z} 1$ & 0.988 & 0.986 \\
& $\mathrm{Z} 2$ & 0.920 & 0.904 \\
\hline
\end{tabular}


Table 5 Results of the ANOVA for the identification of patterns obtained under different conditions. P-values of the condition factor (velocity or gradient) in the result of ANOVA with factors (joints $\times$ conditions) are listed. There are statistically significant differences between subjects at the $1 \%$ level.

(i) P-values for differences due to change in velocity.

DS

SS

\begin{tabular}{cccccc}
\cline { 6 - 6 } \cline { 5 - 6 } Subject & $\mathrm{Z}_{1}$ & $\mathrm{Z} 2$ & & $\mathrm{Z} 1$ & $\mathrm{Z} 2$ \\
\hline $\mathrm{HO}$ & 0.976 & $(<0.01)$ & & $(<0.01)$ & $(<0.01)$ \\
$\mathrm{AD}$ & $(<0.01)$ & 0.212 & & 0.167 & $(<0.05)$ \\
$\mathrm{MA}$ & 0.225 & 0.141 & & $(<0.01)$ & $(<0.01)$ \\
$\mathrm{KI}$ & 0.330 & 0.494 & & $(<0.01)$ & 0.836 \\
$\mathrm{KO}$ & $(<0.05)$ & $(<0.01)$ & 0.098 & $(<0.01)$ \\
$\mathrm{MO}$ & 0.221 & 0.923 & & 0.082 & 0.105 \\
$\mathrm{NA}$ & 0.274 & 0.829 & & $(<0.05)$ & 0.285 \\
$\mathrm{MI}$ & 0.246 & 0.053 & & $(<0.05)$ & $(<0.05)$ \\
$\mathrm{KU}$ & $(<0.01)$ & 0.2701 & & $(<0.01)$ & 0.844 \\
$\mathrm{AN}$ & 0.153 & 0.204 & & 0.163 & 0.500
\end{tabular}

(ii) P-values for differences due to change in incline.

\begin{tabular}{cccccc} 
& \multicolumn{2}{c}{$\mathrm{DS}$} & & \multicolumn{2}{c}{$\mathrm{SS}$} \\
\cline { 2 - 3 } \cline { 5 - 6 } Subject & $\mathrm{Z} 1$ & $\mathrm{Z} 2$ & & $\mathrm{Z} 1$ & $\mathrm{Z} 2$ \\
\hline $\mathrm{HO}$ & 0.059 & $(<0.01)$ & & 0.126 & $(<0.05)$ \\
$\mathrm{AD}$ & $(<0.01)$ & $(<0.05)$ & 0.112 & $(<0.01)$ \\
$\mathrm{MA}$ & 0.293 & $(<0.01)$ & & $(<0.05)$ & $(<0.05)$ \\
$\mathrm{KI}$ & $(<0.01)$ & 0.162 & & $(<0.05)$ & $(<0.05)$ \\
$\mathrm{KO}$ & $(<0.05)$ & 0.094 & 0.379 & $(<0.01)$ \\
$\mathrm{MO}$ & $(<0.01)$ & $(<0.01)$ & 0.789 & $(<0.01)$ \\
$\mathrm{NA}$ & $(<0.01)$ & 0.051 & & $(<0.01)$ & $(<0.05)$ \\
$\mathrm{MI}$ & 0.053 & $(<0.01)$ & $(<0.01)$ & $(<0.01)$ \\
$\mathrm{KU}$ & $(<0.01)$ & $(<0.01)$ & 0.530 & $(<0.05)$ \\
$\mathrm{AN}$ & $(<0.05)$ & $(<0.01)$ & 0.082 & $(<0.01)$ \\
\hline
\end{tabular}

